\title{
Computerized assessment of cognition in schizophrenia: Promises and pitfalls of CANTAB
}

\author{
Marie-Noëlle Levaux ${ }^{\mathrm{a}, \mathrm{c}}$, Stéphane Potvin ${ }^{\mathrm{a}, \mathrm{d}}$, Amir Ali Sepehry ${ }^{\mathrm{a}, \mathrm{b}, \mathrm{d}}$, Juliette Sablier ${ }^{\mathrm{a}, \mathrm{d}}$, Adrianna Mendrek ${ }^{\mathrm{a}, \mathrm{b}}$, \\ Emmanuel Stip a,b \\ ${ }^{a}$ Centre de recherche Fernand-Seguin, Hôpital Louis-H Lafontaine, Montréal, Quebec, Canada \\ ${ }^{\mathrm{b}}$ Department of Psychiatry, Faculty of Medicine, University of Montreal, Montréal, Quebec, Canada \\ ${ }^{\mathrm{c}}$ Department of Psychology, Faculty of Arts and Sciences, University of Montreal, Montréal, Quebec, Canada \\ ${ }^{\mathrm{d}}$ Department of Biomedical Sciences, Faculty of Medicine, University of Montreal, Montréal, Quebec, Canada
}

\begin{abstract}
Objective. - Over the last decade, the Cambridge Neuropsychological Test Automated Battery (CANTAB), which comprises visuo-spatial tasks, has been utilized in cognitive studies of schizophrenia. A clear approach concerning the usage of CANTAB for the appraisal of neuro-cognitive dysfunction in schizophrenia is currently lacking.
\end{abstract}

Method. - In this paper, we have first reviewed the overall applications of CANTAB and then evaluated methodological strengths and weaknesses of CANTAB as a neurocognitive battery for schizophrenia. We carried out a systematic search and assessment of studies where CANTAB was utilized to measure cognitive function in schizophrenia. We have also attempted to quantify the available data and perform a meta-analysis, but this approach turned out to be still premature.

Results. - CANTAB has enabled researchers to highlight significant deficits affecting broad cognitive domains in schizophrenia, such as working memory, decision-making, attention, executive functions and visual memory. So far, the most consistent deficit observed with CANTAB testing has been attentional set-shifting, suggestive of fronto-striatal dysfunctions. In addition, preliminary evidence points towards the potential use of CANTAB to identify cognitive predictors of psychosocial functioning, to describe the relationships between symptoms and cognition, and to measure the impact of pharmacological agents on cognitive functioning.

Conclusion. - CANTAB has been used successfully to highlight the range of visuo-spatial cognitive deficits in schizophrenia, producing similar results to those obtained with some traditional neuropsychological tests. Further studies validating the use of CANTAB following the standard set by Measurement And Treatment Research to Improve Cognition in Schizophrenia (MATRICS) are warranted.

Keywords : Schizophrenia ; Cognition ; CANTAB ; Memory

\section{Introduction}

Over the last decade, the Cambridge Neuropsychological Test Automated Battery (CANTAB) has been utilized in cognitive studies of schizophrenia. The CANTAB was originally written and developed at the University of Cambridge by Barbara Sahakian, Trevor Robbins and colleagues in 1986. It consists of a series of interrelated computerized non-verbal tests of memory, attention, and executive function. At the present time CANTAB consists of 19 visuo-spatial tests (CANTAB eclipse version 2.0). The tests are: Affective Go/ No-go (AGN); Big/Little Circle (BLC); Cambridge Gambling Task (CGT); Choice Reaction Time (CRT); Delayed Matching to Sample (DMS); Intra-Extra Dimensional Set Shifting (IED); Graded Naming Test (GNT); Matching to Sample Visual Search (MTS); Motor Screening (MOT); Paired Associated Learning (PAL); Pattern Recognition Memory (PRM); Rapid Image Visual Processing (RVP); Reaction Time (RTI); Spatial Recognition Memory (SRM); Spatial Span (SSP); Spatial Working Memory (SWM); Stocking of Cambridge (SOC); Simple Reaction Time (SRT); Verbal Recognition Memory (VRM). For more details, see Table 1 and http://www.cantab.com/cantab/site/home.acds. IED, SOC, SSP and RVP tests are respectively close versions of 
the Wisconsin Card Sorting Test [8], the Tower of London [60], the Corsi's Block Tapping Task (CBT) [36] and the Continuous Performance Test (CPT) [56].

In this review, we first discuss the methodological strengths and weaknesses of CANTAB as a neurocognitive battery. Then, we evaluate various applications of CANTAB in schizophrenia, which has been utilized to highlight cognitive deficits in schizophrenia (compared to healthy controls and other patient populations), to identify cognitive predictors of the disease, to identify cognitive predictors of psychosocial functioning, to divide schizophrenia into subgroups with enhanced phenotypic homogeneity, to describe the relationships between cognitive and occulomotor deficits in schizophrenia and to measure the impact of pharmacological agents on cognition (Table 2).

Table 1 Scales description

\begin{tabular}{|c|c|c|}
\hline$\overline{\mathrm{AGN}}$ & Affective Go/No-go & $\begin{array}{l}\text { Information processing biases for positive and negative stimuli } \rightarrow \text { mean } \\
\text { correct latency/total commissions/ total omissions. Not language free }\end{array}$ \\
\hline BLC & Big/Little Circle & $\begin{array}{l}\text { Comprehension and learning reversal } \rightarrow \text { percent correct/total } \\
\text { correct/total attempts/total errors/mean correct latency }\end{array}$ \\
\hline CGT & Cambridge Gambling Task & $\begin{array}{l}\text { Impulse control and risk-taking in decision making } \rightarrow \text { quality of } \\
\text { decision making/deliberation time/risk taking/risk adjustment/delay } \\
\text { aversion/overall proportion bet }\end{array}$ \\
\hline CRT & Choice Reaction Time & $\begin{array}{l}\text { Speed of choice response } \rightarrow \text { latency/correct responses/incorrect } \\
\text { responses/omissions/commissions }\end{array}$ \\
\hline DMS & Delayed Matching to Sample & $\begin{array}{l}\text { Simultaneous and delayed perceptual matching } \rightarrow \text { numbers and } \\
\text { percentage correct/latency/statistical analysis }\end{array}$ \\
\hline GNT & Graded Naming Test & $\begin{array}{l}\text { Lexical/semantic memory by assessing object-naming ability } \rightarrow \text { total } \\
\text { correct/total errors/totals attempts/ percent correct/normative } \mathrm{Z} \text { - } \\
\text { score/normative percentile }\end{array}$ \\
\hline IED & $\begin{array}{l}\text { Intra-Extra Dimensional Set } \\
\text { Shifting }\end{array}$ & $\begin{array}{l}\text { Rule acquisition and attentional set-shifting } \rightarrow \text { errors/original } \\
\text { focus/numbers of trials and stage completed }\end{array}$ \\
\hline MOT & Motor Screening & $\begin{array}{l}\text { Visuo-motrice coordination and comprehension difficulties } \rightarrow \text { mean } \\
\text { latency/mean error }\end{array}$ \\
\hline $\begin{array}{l}\text { MTS- } \\
\text { VS }\end{array}$ & $\begin{array}{l}\text { Matching to Sample Visual } \\
\text { Research }\end{array}$ & $\begin{array}{l}\text { Ability to match visual samples, reaction time and movement time } \rightarrow \\
\text { correct numbers/correct percentages/ latency }\end{array}$ \\
\hline PAL & Paired Associates Learning & $\begin{array}{l}\text { Episodic visuo-spatial memory, learning and association ability } \rightarrow \\
\text { errors/trials/stages completed/memory scores }\end{array}$ \\
\hline PRM & Pattern Recognition Memory & $\begin{array}{l}\text { Recognition memory for patterns } \rightarrow \text { numbers and percentages correct } \\
\text { and incorrect/latency }\end{array}$ \\
\hline RTI & Reaction Time & Speed of response $\rightarrow$ reaction time/movement time \\
\hline RVP & $\begin{array}{l}\text { Rapid Visual Information } \\
\text { Processing }\end{array}$ & $\begin{array}{l}\text { Sustained visual attention } \rightarrow \text { hits, misses, false alarm and } \\
\text { rejections/probabilities and sensitivity calculated using SDT/latency }\end{array}$ \\
\hline $\mathrm{SOC}$ & Stockings of Cambridge & $\begin{array}{l}\text { Spatial planning and motor control } \rightarrow \text { problem solved in minimum } \\
\text { moves/initial thinking time for } 2,3,4 \text {, and } 5 \text {-move problems/subsequent } \\
\text { thinking time for } 2,3,4 \text {, and } 5 \text {-move problems/mean moves for } 2,3,4 \text {, } \\
\text { and 5-move problems. NTOL: adaptation of the previous task with } \\
\text { reducing the motors demands placed on subject }\end{array}$ \\
\hline SRM & Spatial Recognition Memory & $\begin{array}{l}\text { Recognition memory for spatial locations } \rightarrow \text { number correct/percent } \\
\text { correct/mean correct latency }\end{array}$ \\
\hline SRT & Simple Reaction Time & $\begin{array}{l}\text { Speed of response to a single stimulus } \rightarrow \text { correct } \\
\text { responses/commissions/omissions/latency }\end{array}$ \\
\hline SSP & Spatial Span & Working memory capacity $\rightarrow$ span length/errors \\
\hline SWM & Spatial Working Memory & $\begin{array}{l}\text { Working visuo-spatial memory and strategy use } \rightarrow \text { errors (between, } \\
\text { within, double and total)/strategy score }\end{array}$ \\
\hline VRM & Verbal Recognition Memory & $\begin{array}{l}\text { Immediate free recall, and immediate and delayed recognition memory } \\
\rightarrow \text { free recall: total correct, total novel words, total } \\
\text { perseverations/recognition: total correct, total false positives. Not } \\
\text { language free }\end{array}$ \\
\hline
\end{tabular}


Table 2 Study demographics

\begin{tabular}{|c|c|c|c|c|}
\hline $\begin{array}{l}\text { Study } \\
\text { references }\end{array}$ & Test items & Drug Tx. & $N$ & Subjects diagnosis \\
\hline$[1,2]$ & SSP, DMS, SWM, IED, SOC & Medicated & 47 & Paranoid \\
\hline$[3]$ & SSP, SWM, SOC & Medicated & 24 & Schizophrenia/inpatient \\
\hline$[5]$ & PRM, SRM, SOC, IED & Mixed & 53 & First-episode \\
\hline$[6]$ & SSP, PRM, SOC, SWM & Medicated & 152 & First-episode \\
\hline$[7]$ & $\begin{array}{l}\text { MOT, BLC, SWM, RTI, SSP, PRM, SRM, RVP, } \\
\text { PAL, IED, MTS, DMS, SOC }\end{array}$ & Mixed & 11 & Pre-psychotic \\
\hline$[26]$ & SOC, SWM, SSP, IED, PRM, SRM & Medicated & 136 & First-episode \\
\hline$[27]$ & SSP, PRM, SWM, SOC, IED & Medicated & 93 & First-episode \\
\hline$[16]$ & IED, DMS, PRM, SRM, PAL, SSP, SWM & NA & 28 & Elder schizophrenia \\
\hline$[30]$ & SSP & Medicated & 40 & Chronic \\
\hline$[33]$ & DMS & Medicated & 105 & $\begin{array}{l}\text { First- } \\
\text { episode/schizophreniform/ } \\
\text { schizophrenia }\end{array}$ \\
\hline$[35]$ & SOC, SRT, CRT & Medicated & 27 & Schizophrenia \\
\hline$[43]$ & MOT, SWM, SOC, SSP & Medicated & 36 & Chronic/inpatient \\
\hline$[42]$ & IED & Medicated & 24 & Chronic/inpatient \\
\hline$[44]$ & SSP, SWM, PRM, SRM, DMS & Medicated & 54 & Chronic/inpatient \\
\hline [45] & PAL, MOT & Medicated & 76 & DD/outpatient \\
\hline$[48]$ & SOC, RTI, PAL, MOT & Medicated & 88 & Outpatient \\
\hline$[50]$ & SOC, RVP, RTI, PAL, MOT & Medicated & 73 & Outpatient \\
\hline$[49]$ & MOT, PAL, RTI, SOC, RVP & Medicated & 55 & Outpatient \\
\hline [10] & IED, PRM, SRM & Medicated & 32 & Chronic \\
\hline$[11]$ & PRM, SRM, DMS, SSP, SWM, SOC, IED, NTOL & Medicated & 12 & Chronic \\
\hline$[52]$ & $\begin{array}{l}\text { MOT, RTI, MTS, DMS, PRM, SRM, PAL, SSP, } \\
\text { RVP, SWM, IED, SOC }\end{array}$ & Medicated & 55 & $\begin{array}{l}\text { Schizophrenia/schizo- } \\
\text { affective out/inpatient }\end{array}$ \\
\hline$[25]$ & SSP, PRM, SRM, SWM, SOC, IED & Mixed & 30 & First-episode \\
\hline [22] & SWM, SSP, PRM, SRM & Mixed & 63 & First-episode \\
\hline$[24]$ & CGT & Medicated & 50 & First-episode/chronic \\
\hline [21] & SSP, PRM, SWM, SOC, IED & Medicated & 109 & First-episode \\
\hline$[62]$ & SOC, MOT, PAL, RTI & Medicated & 57 & $\begin{array}{l}\text { Schizophrenia/schizo- } \\
\text { affective outpatient }\end{array}$ \\
\hline$[64]$ & MOT, PRM, DMS, SWM, SSP, NTOL, IED & Medicated & 20 & Chronic \\
\hline$[66]$ & IED, SOC & Medicated & 28 & Out/inpatient \\
\hline [65] & PRM, SRM, SSP, SWM & Medicated & 28 & Out/inpatient \\
\hline$[67]$ & SSP, SWM, DMS & NA & 38 & High risk \\
\hline
\end{tabular}

NA, not available; DD, Dual Diagnosis.

\section{CANTAB defined}

The development of the CANTAB was heavily guided by a number of existing neuropsychological paradigms commonly used to assess cognitive dysfunction in non-human primates with selective neurochemical or neuroanatomical lesions. The developers adapted experimental tasks with longstanding traditions of brain-tobehavior assessment into a format that could be readily accessed by human participant with neurological or psychiatric disorders, or both [15]. However, as the battery was developed, tests based on methodological advances in neuropsychological assessment of human patients were also added, for example, a DMS procedure analogous to the one used by Gaffan [17] and Mishkin [37] to determine the neural basis of visual recognition memory in infrahuman primates [61]. Individual tasks have been extensively studied in order to define and validate their neural correlates, particularly with the goal of dissociating the performance of humans with frontal versus temporal lobe neuropathology [38-40,54] and to distinguish patterns of cognitive deficits in temporal versus frontal-lobe dementias [28,58,59]. Robbins and collaborators [53] have administered the CANTAB battery to a large group of elderly volunteers in the age range of 55-80 years and showed that scores on CANTAB subtests fall into readily separable factors which can plausibly be identified with particular groups of cognitive functions that are known to be supported by distinct neural subsystems. For example, the SWM is especially dependent upon intact frontal lobes, in contrast to the PRM which is unaffected by frontal damage but is sensitive to lesions of the temporal lobe [40]. In a positron emission tomography (PET) scanning study, Baker 
and colleagues [4] found that for the SOC, a task analogue to the Tower of London test [60], there is widespread activation of the parietal and occipital cortices, and other distinct areas of the frontal lobe, including the anterior cingulate cortex which is associated with task difficulty. Most importantly, they have demonstrated no differential temporal lobe activation, confirming the fact that this task implicates neural pathways distinct from the DMS, which only involves the temporal lobe, but not the frontal cortex and its associated frontostriatal loops [4].

CANTAB has been standardized on a large population aged between 4 and 90 years old in various research studies [63], and its validity has been established in a wide variety of clinical populations [15], including neurodegenerative diseases, neurosurgical cases, psychiatric disorders, and acquired pathology. Some of the specific disorders include: Alzheimer's disease [58,59], dementia of the frontal type [57], Parkinson's disease (PD) [58], Huntington's disease [20], frontal, temporal lobe and amygdalo-hippocampectomy [40], and unipolar depression [12]. Neurological validation may enable specific neurobiological hypotheses to be addressed. Furthermore, these tests have been shown to possess acceptable to high levels of concurrent validity and testretest reliability [14].

\subsection{Strengths and weaknesses of CANTAB}

CANTAB has advantages and some disadvantages, mostly related to its practical application and methodological concerns, which are described in Table 3.

Table 3 Strengths and weaknesses

\begin{tabular}{ll} 
Strengths \\
\hline 1 Standardized testing procedure \\
2 User-friendly quality
\end{tabular}

2 User-friendly quality

3 Can be administered by a nonneuropsychologist

4 Non-verbal nature

5 Flexible batteries

6 Graded nature

Weaknesses

1 Relatively expensive

2 Mainly visuo-spatial cognitive functions

3 Lack of comparison to traditional neuropsychological tests

4 Questionable test/re-test reliability and practice effects
Includes standardized feedback and detailed recording of data

Touch screen monitor

Can continuously be checked to ensure that task demands are correctly understood and

levels of difficulty can continuously be adjusted

Playful, attractive interface and game-like quality

Nonetheless, basic knowledge in neuropsychological assessment is required

Largely language-independent and culture-free, permitting multicentre studies

Allows selecting tasks specific to research or clinical interests, using clinical or parallel

versions of the test

Parallel version of the task permit for testing in repeated measure designs [64]

Reduces the likelihood of floor and ceilings effects

Purchasing a touch screen monitor

Limits conclusions about patient's verbal functioning

Exceptions are: Verbal Recognition Memory (VRM) and Graded

Naming Test (GNT)

Difficult to produce an interpretation of results in line with traditional neuropsychological concepts, models and theories Lowe and Rabbit [31] have evaluated test/re-test correlations and practice effects for CANTAB from large groups of healthy elderly and found many of the CANTAB measures have poor test-retest reliability and modest practice effects

Table 4 Frequencies in subscale usage

\begin{tabular}{llll}
\hline Scales & Name & Frequency $(\%)$ & Study references \\
\hline CGT & Cambridge Gambling Task & $1 / 31(3.23)$ & {$[24]$} \\
CRT & Choice Reaction Time & $1 / 31(3.23)$ & {$[35]$} \\
DMS & Delayed Matching to Sample & $10 / 31(32.26)$ & {$[1,2,7,11,16,33,44,52,64,67]$} \\
IED & Intra/extra-Dimensional Set Shifting & $14 / 31(43.16)$ & {$[1,5,7,10,11,16,21,25-27,42,52,64,66]$} \\
MOT & Motor Screening & $8 / 31(25.8)$ & {$[7,43,46,49,50,52,62,64]$}
\end{tabular}




\begin{tabular}{llll} 
MTS & Matching to Sample Visual Search & $2 / 31(6.45)$ & {$[7,52]$} \\
OTS & One Touch & $2 / 31(6.45)$ & {$[11,64]$} \\
& Stockings of Cambridge & & \\
PAL & Paired Associates Learning & $8 / 31(25.8)$ & {$[7,16,46,48-50,52,62]$} \\
PRM & Pattern Recognition Memory & $14 / 31(43.16)$ & {$[5,7,10,11,16,21,22,25-27,30,44,52,64]$} \\
RTI & Reaction Time & $6 / 31(19.35)$ & {$[7,48-50,52,62]$} \\
RVP & Rapid Visual Processing & $4 / 31(12.9)$ & {$[7,48,49,52]$} \\
SOC & Stockings of Cambridge & $17 / 31(54.84)$ & {$[1,3,5-7,11,21,25-27,35,48-50,52,62,66]$} \\
SRM & Spatial Recognition Memory & $12 / 31(38.7)$ & {$[5,10,11,16,21,22,25,30,44,52,64,65]$} \\
SRT & Simple Reaction Time & $1 / 31(3.23)$ & {$[35]$} \\
SSP & Spatial Span & $17 / 31(54.84)$ & {$[1,3,6,7,11,16,22,23,26,27,43,44,52,64,6$} \\
& & & $5,67]$ \\
SWM & Spatial Working Memory & $16 / 31(51.61)$ & {$[1,3,6,7,11,16,21,22,25-$} \\
& & & $27,43,52,64,65,67]$ \\
\hline
\end{tabular}

\section{CANTAB in schizophrenia}

\subsection{Cognitive deficits in schizophrenia}

The tests of CANTAB used in studies of schizophrenia have allowed examination of cognitive functions such as attention, memory and executive functions, characterizing the nature of the impairment and the association with focal brain regions. After a compilation of all the studies investigating cognition in schizophrenia using CANTAB, we have tabulated a frequency table of the tasks utilized to date (Table 4). The most frequent tasks were as follow: SOC (13 studies); SWM (10 studies); PRM (10 studies), SRM (10 studies); IED (11 studies). This literature has highlighted broad cognitive deficits in schizophrenia, involving short-term memory, long-term memory, attention, and executive functions.

\subsection{Schizophrenia patients}

Elliott et al. [10] compared the performance of patients suffering from schizophrenia to controls on the IED task. This task provides a componential analysis of the Wisconsin Card Sorting Test (conditions termed 'perseveration' and 'learned irrelevance') and it was earlier revealed to be sensitive to frontal lobe dysfunction and PD. Medicated chronic schizophrenia patients proved to be significantly impaired on the perseveration but not the learned irrelevance condition. This result points to specific deficits in set-shifting ability of executive function in schizophrenia. Tyson et al. [66] investigated executive functions involving attentional set-shifting tested with IED and planning abilities tested with SOC in schizophrenia patients. They found that on both tests, patients performed poorer than controls. The authors also tested whether these deficits are stable during the course of the illness (trait deficits) or if they fluctuate along symptom changes (state deficits). They observed no change in cognitive performance during the 9-month follow-up, a result consistent with the hypothesis that executive dysfunctions in schizophrenia are stable over time. Almeida et al. [1] investigated cognitive functioning in psychotic states arising in late life (late paraphrenia) using the IED, SWM and SOC tasks. Compared to controls, patients showed deficits in all tasks. Based on these findings, the authors proposed that late paraphrenia is linked with a decline in measures of universal abilities and executive skills, instead of a distinctive pattern of cognitive impairments. Tyson et al. [65] investigated memory deficits in a schizophrenia group with a wide range of illness length (3 weeks to 38 years), using the PRM, SSP and SWM tasks. They showed that patients with schizophrenia have deficits in all aspects of memory measured with CANTAB, and that these deficits remain stable over time (at least over 18 months). The above studies highlight broad cognitive deficits in schizophrenia, especially deficits on the IED task.

\subsection{First-episode schizophrenia patients}

Hutton and Huddy [21] studied cognitive functioning in first-episode schizophrenia patients, using the SSP, PRM, SWM, SOC and IED tasks. They observed deficits on all these tasks in patients, relative to controls. Joyce et al. [26] investigated executive functions in first-episode schizophrenia patients and found that SWM was impaired in patients because of inadequate strategy use. Patients also showed reduced planning time and suboptimal problem-solving ability when tested with SOC. However, it must be considered that Hutton et al. [25] were not able to replicate the finding of an impaired performance of first-episode schizophrenic patients on the IED task, as shown by Tyson et al. [66], Almeida et al. [1], Hutton and Huddy [21], and Elliott et al. [10]. 
Examining executive function (planning and strategy) in first-episode patients with tests known to be sensitive to frontal lobe function (e.g. SOC, SWM, and IED), Hutton et al. [25] showed that patients suffering from schizophrenia have profound executive impairments, but not in the attentional set-shifting abilities, which were generally unimpaired.

Employing the DMS task in first-episode schizophreniform disorder and established schizophrenia, Mathes et al. [33] have disentangled impairments of working memory subprocesses. A deficit was found in both patient groups in the processes essential to actively maintain information.

Using a task sensitive to orbitofrontal dysfunction, the newly developed CGT, Hutton et al. [24] measured decision making in first-episode and chronic schizophrenia patients. The deficits highlighted using this task were more prominent in the chronic patients, relative to the first-episode patients. This finding further adds to the already growing evidence of orbitofrontal dysfunction in schizophrenia, and advises that the evolution of the disease or long duration of treatment with antipsychotics may actually sway performance on this task.

\subsection{Subtyping schizophrenia's cognitive profiles}

Considering the symptomatic heterogeneity of schizophrenia, important efforts have been invested in providing a more refined description of the phenotypic features of the pathology, using experimental procedures, including neuropsychological tests [27]. From that perspective, CANTAB tasks have been recently used to divide schizophrenia patients into distinctive subgroups according to cognitive profiles, both empirically and clinically useful.

A study by Almeida et al. [2] has explored the heterogeneity of late paraphrenia, using the DMS, IED, SSP, and SWM tasks. The authors performed a factor analysis which produced a division of paranoid psychosis into two patient clusters: type A, which corresponds to patients with a wide range of psychotic symptoms, mild neurological signs, and cognitive deficits restricted to executive functions; and type B, which includes patients with less complex psychotic symptoms associated with marked neurological signs and generalized cognitive impairments.

Joyce et al. [27] with utilization of SSP, PRM, SWM, SOC, and IED tasks in first-episode schizophrenia patients, determined cognitive heterogeneity in chronic schizophrenia to be also present at illness onset. They have found that patients who demonstrated preserved IQ in the normal range achieved similar scores to the controls, whereas other patients showed a decline in neuropsychological function. On the contrary, impairment of the spatial working memory was seen as a common feature in all patients.

Potvin et al. [45] used the MOT and PAL tasks to compare the cognitive performance of schizophrenia patients with and without a substance use disorder (SUD), since the lifetime prevalence of SUD is close to $50 \%$ in schizophrenia [51]. The authors found that dual diagnosis patients showed a better performance. They concluded that their finding suggests either that substance abuse relieves the cognitive deficits of schizophrenia or that the patients with less cognitive deficits are more prone to substance abuse. However, Barnes et al. [6] also compared the cognitive functioning of single and dual diagnosis patients, using CANTAB tasks (PRM, SOC, SSP, and $\mathrm{SWM}$ ) and found no significant differences between the two groups.

In a two-year longitudinal study, Stip et al. [62] used CANTAB tasks (e.g. MOT, SOC, PAL, and RTI) as cognitive discernable factors between schizophrenia and schizoaffective disorder. They found that the PAL and MOT, but not the SOC and RTI, discriminated between the two patient groups, as patients with schizoaffective disorder performed better in time on these two cognitive tasks. These results suggest that visuo-spatial explicit memory and visuo-motor coordination may be relevant for distinguishing schizophrenia from schizoaffective disorder.

Although encouraging, the results from these studies must be replicated by other research teams before it can be concluded that CANTAB is useful for schizophrenia's cognitive profile subtyping.

\subsection{Predictors of schizophrenia}

Studies have been performed to identify behavioral and cognitive predictors of psychosis [3,67]. These studies are important since they may help identify subjects who are psychosis-prone and elaborate programs to prevent the development of psychosis. 
Preliminary evidence suggests that CANTAB may be a useful tool to identify cognitive dysfunctions predicting psychosis prior to its apparition. For instance, Wood et al. [67] have investigated whether working memory and executive functions as tested with the SWM and DMS tasks were impaired in young people at ultrahigh risk of developing psychosis. Both SWM and DMS performance was poorer in the ultrahigh risk subjects relative to controls. A non-significant trend emerged towards poorer cognitive performance among subjects who later became psychotic compared to those who did not. Those findings suggest that CANTAB could be used as a predictive tool to detect subjects at risk of developing psychosis. Similarly, Bartok et al. [7] have investigated memory and attention in young adults at the prodromal stage of psychosis, using the PAL, SRM, RVP, and SWM tasks. They observed deficits on all tasks in the pre-psychotic patients, relative to healthy controls. Further studies to validate the use of CANTAB as a predictive tool for early detection of individuals at risk of developing psychosis are warranted.

\subsection{Community functioning}

Since neuropsychological tests do not reflect real-life situations, concerns have been raised about their ecological validity. CANTAB faces the same problem as traditional neuropsychological tests. To address this problem, Prouteau et al. [48] have used CANTAB to explore the pattern of associations between visuo-spatial cognition and community functioning in schizophrenia outpatients participating in a rehabilitation program (15-16 month follow-up). CANTAB MOT, PAL, RTI, RVP, and SOC were administered, and community functioning was measured with the Multinomah Community Ability Scale (assessed on baseline and endpoint). Using multiple regression analyses, they found that a better sustained attention performance was associated with better global community functioning at baseline. In a subsequent study involving the same participants, Prouteau et al. [49] were further able to show that better baseline visual memory (PAL) predicted better community functioning at endpoint (e.g. greater daily living autonomy and greater social competence). Moreover, baseline sustained attention performance (RVP) predicted improvements in behavioral problems (e.g. medication compliance) and social competence. Planning abilities (SOC) also predicted improvements in social competence.

The same group [50] also explored the pattern of associations between self-assessed and objective neuropsychological performance, using CANTAB and the Subjective Scale to Investigate Cognition in Schizophrenia (SSTICS). They found an association between higher subjective complaints and poorer CANTAB explicit visual memory (PAL) and planning (SOC) performances. Potvin et al. [45] found similar associations in dual diagnosis patients, using the PAL and the SSTICS.

Overall, the fact that cognition, as measured with CANTAB, predicted psychosocial functioning is consistent with the results obtained in studies using traditional neuropsychological tests [18]. Further studies are needed to validate the use of CANTAB to identify cognitive predictors of social functioning.

\subsection{Relationships between cognitive and occulomotor functions}

Schizophrenia is associated with subtle occulomotor deficits, which are positively correlated with cognitive impairments, mostly frontal lobe deficits $[21,22]$. Although the topic has not been systematically investigated, a few studies have explored the relationships between occulomotor and cognitive deficits in schizophrenia using CANTAB. For instance, Hutton et al. [22] investigated the likelihood of smaller (hypometric) saccades in firstepisode schizophrenia patients (relative to controls), and whether hypometria would mirror spatial memory impairments (using SSP, SWM SRM, and PRM tasks). They found that schizophrenia patients (both drug-naive and treated) made hypometric saccades, relative to healthy controls. In addition, they found that primary saccade correlated with SRM, PRM, and SMW scores.

Hutton and Huddy [21] investigated, in first-episode schizophrenia patients, the relationships between cognitive impairments (measured with the SSP, PRM, SWM, SOC, and IED tasks) and occulomotor deficits measured using smooth pursuit and antisaccade performance tasks. The authors found deficits in smooth pursuit and antisaccade performance in patients, relative to controls. Also, they noticed a correlation between antisaccade errors and spatial working memory performance, but no correlation between smooth pursuit velocity and cognition. Based on this finding, the authors proposed that spatial working memory and antisaccade performance may share the same prefrontal pathways in schizophrenia.

Although preliminary, the relationships described in these studies between occulomotor and cognitive deficits in schizophrenia are consistent with the results of studies using traditional neuropsychological tests [29].

In a related domain, it must be noted that Pantelis et al. [44] studied the relationships between negative 
symptoms, orofacial tardive dyskinesia (OTD) and memory and executive functions. Using a components analysis, they found that OTD and negative symptoms were independently associated with spatial working memory deficits in schizophrenia.

\subsection{Schizophrenia vs. other disorders and acquired pathology}

CANTAB has been used in many papers to compare the cognitive impairments of schizophrenia to those of other patient groups. Allowing a description of the distinctive cognitive deficits of schizophrenia, these studies have also an obvious pathophysiological underlying relevance.

Using the IED task, Pantelis et al. [42] evaluated patients suffering from schizophrenia (chronic), patients with frontal lobe lesion, and a control group. By means of a component analysis of the Wisconsin Card Sorting Test (WCST), the IED task has been designed and it comprises two types of set-shifts: (i) "intra-dimensional shifts (IDS), which involve the transfer of a rule within the same stimulus dimension (e.g. choosing circles instead of squares); and (ii) extra-dimensional shifts (EDS), which require a transfer of attention across different stimulus dimensions (e.g. choosing on the basis of color rather than the previous category of shape)" [42]. Compared to controls and frontal lobe lesion patients, schizophrenia patients had a poorer performance at the intradimensional shift stage of learning, suggesting a failure of inhibitory mechanisms. At the extra-dimensional shift stage, schizophrenia patients with prominent negative symptoms exhibited comparable deficits to the frontal lobe lesion group. These results were interpreted by the authors as being consistent with the notion of separate pathophysiological mechanisms in schizophrenia, involving separate fronto-striatal systems [55].

Using the MOT, SSP, WSM, and SOC tasks, Pantelis et al. [43] have assessed spatial working memory, spatial span, motor speed and planning abilities in patients with chronic schizophrenia, normal subjects and patients with neurological disorders (frontal lobe, temporal lobe and amygdalo-hippocampal lesions, PD). The results of this study are indicative of an overall deficit of executive functioning in schizophrenia, even greater than that seen in patients with frontal lobe lesions. In addition, the pattern of results (deficits in SWM and SOC) in schizophrenia resembled that seen in patients with lesions of the frontal lobe or with basal ganglia dysfunction and, to a lesser extent, similar to those found in patients with PD. In contrast, there were no similarities on any of the administered tests between the performance of the patients with schizophrenia and that of patients with focal temporal lobe lesions. These results provide support to the notion of disturbances in fronto-striatal circuits in schizophrenia.

Using numerous CANTAB tasks (e.g. IED, DMS, PRM, SRM, PAL, SSP, and SWM), Gabrovska-Johnson et al. [16] attempted to characterize the neuropsychological deficits in elderly schizophrenia patients and distinguish them from those in Alzheimer's disease (AD) and to establish the presence of underlying structural brain abnormalities using functional magnetic resonance imaging (fMRI). They found that schizophrenia patients were more impaired on most visuo-spatial tasks than $\mathrm{AD}$ patients but less impaired on corresponding verbal tasks, despite similar overall cognitive impairment. Moreover, the MR scans revealed a correlation between impaired performance on visuo-spatial tasks and right-sided enlargement of ventral cerebrospinal fluid (CSF) spaces in the schizophrenia patients.

Using a wide range of cognitive tests, including the CANTAB SOC task, Mcintosh et al. [35] examined whether cognitive abnormalities are related to a genetic liability to schizophrenia. Patients suffering from schizophrenia and bipolar disorder performed worse on the SOC than controls. Unaffected relatives from "schizophrenia" and "mixed bipolar disorder" families also performed on the task. However, these differences did not remain significant after adjustment for intellectual function.

Badcock et al. [3] have examined the specificity of the spatial working memory impairments by comparing the mnemonic and executive aspects of working memory performance in schizophrenia and bipolar disorder with psychotic features. Both patient groups were impaired relative to the control group on the spatial span task; which requires the maintenance and retrieval of stored information. In contrast, only schizophrenia patients showed a significant deficit on a SWM sub-score (e.g. "between search errors"), which requires both maintenance and manipulation of information in working memory. That is, schizophrenia patients exhibited deficits in both short-term memory and executive functions, whereas bipolar I patients displayed only mnemonic dysfunctions. Based on this finding, the authors evoked a possible common disturbance in fronto-parietal circuitry in the two disorders together with a specific disturbance of fronto-striatal circuitry in schizophrenia.

In the study of Elliott et al. [11], patients with unipolar depression showed similar deficits to those observed in schizophrenia patients when tested with DMS but not with IED or the SOC. Again, these results are consistent 
with the findings of both mnemonic and executive dysfunctions in schizophrenia.

Studies comparing schizophrenia patients to other patient groups using CANTAB have consistently highlighted deficits in executive functions, coherent with fronto-striatal dysfunctions in the disorder. Functional brain imaging studies are required to corroborate this pathophysiological assumption.

\subsection{Pharmacological treatment}

The study of the impact of treatment on cognition in schizophrenia is of importance since cognitive deficits seem to predict psychosocial functioning, as mentioned previously. By improving cognition, a better psychosocial functioning may be expected. Also highlighting the importance of treatment, two studies have used CANTAB and showed a relationship between duration of untreated psychosis (DUP) and cognitive performance (see Table $5)$.

More work is needed before a firm conclusion can be reached about the use of CANTAB to measure cognition in pharmacological studies in schizophrenia. Although, Measurement and Treatment Research to Improve Cognition in Schizophrenia (MATRICS) was developed in order to provide a consensus about neurocognitive tests to be used in schizophrenia and the design of pharmacological trials in this field [19], further studies validating the use of CANTAB in this vein are recommended. Future studies will need to determine the time span between assessments required to avoid practice effects. A direct comparison between clinical and parallel versions is also needed.

\section{Quantitative systematic review method}

In order to present a more systematic review, we tried to consider quantifying the data and thus offer a metaanalysis of CANTAB findings in schizophrenia. This initiative is described but is too premature in terms of results. Searches using electronic search engines, and CANTAB website's bibliographic list were carried out (http:/www.cantab.com/cantab/ site/home.acds?context= 1306318\&instanceid= 1306319) with the following key words: "schizophrenia" AND "CANTAB OR Cambridge Neuropsychological Test Automated Battery". Controlling for the quality of studies, we have included all papers presenting original data of patients suffering from schizophrenia-spectrum disorder compared to healthy volunteers (control), and being tested for cognitive function with CANTAB. A total of 12 cross-sectional studies matched our criteria.

Consistently available test scores across studies were SOC, IED, SSP, DMS, PRM, SWM, and SRM. The number of papers reporting all subscales to a test were limited. As reported by Strauss et al. [63], those tests have adequate, marginal or low magnitude on test-retest coefficient (e.g. IED, SOC, and SRM, respectively) with the exception of PRM, and sometimes subscales of the test evaluation on this coefficient are not there (e.g. SWMstrategy). Yet, in this quantitative approach to cross-sectional studies, the test-retest qualities of the scales remain simply a weakness to the tests and not to the meta-analytic investigation.

Among studies, the psychopathological assessment had few discrepancies. First, the assessment was heterogeneous (e.g. PANSS, SANS or SAPS). Second, not all studies reported symptomatology assessment. Moreover, the medication regimen of the patients involved in those studies is not considered consistent. For example, few studies report on medication type, or do not report them at all.

These three factors, limited number of subscales to tests, and lack of knowledge on symptomatology and medication regimen of the patients, would render investigation, in the event of heterogeneity, rather difficult. Moreover, drawing a conclusion on a set of heterogeneous effect estimate with a limited number of studies enrolled, would reach an inconclusive statement. 
Table 5 Summary of pharmacological studies with CANTAB

\begin{tabular}{lll}
\hline Studies & Patients & Design/procedure \\
\hline Lawrie et al. 1995 [30] & Treatment-resistant and & Functional magnetic resonance \\
& $\begin{array}{l}\text { treatment-responsive } \\
\text { schizophrenia }\end{array}$ & imaging (fMRI); single photon \\
& & emission tomography (SPECT); \\
& investigated biological \\
& abnormalities; controlling for years \\
& of education
\end{tabular}

Results

No broad differences in brain-imaging parameters between the two groups; lower volumes of most brain structures in treatmentresistant patients; treatmentresistant patients displayed a poorer performance on overall set of cognitive tasks (including CANTAB SRM task)

Barnes et al. 2000 [5] $\quad$ First-episode schizophrenia

Examined cognition with CANTAB Patients with long DUP in patients with short and long DUP tended to perform worse on an executive attentional setshifting task, relative to patients with short DUP

Measured the impact of Chlorpromazine enhanced

McCartan et al. 2001 [34] Healthy volunteers chlorpromazine and haloperidol on LI; measures of frontal lobe function were performed with LI, whereas haloperidol was associated with impaired CANTAB

Joyce et al. 2002 [26] First-episode schizophrenia

Investigated the relationship between cognitive (executive dysfunction) performance and DUP SWM

$75 \%$ of patients were able to perform the IED task; $25 \%$ who failed had significantly longer DUP

Turner et al. 2004 [64] Chronic schizophrenia

Chouinard et al. 2005 [9] Schizophrenia

Polypharmacy with cognitive enhancing agent, rivastigmine; cross-over clinical trial; measured cognitive performance

Double-blind, randomized, placebo- Significant improvements in controlled, crossover study; examined the potential cognitive enhancing effect of modafinil attentional set-shifting; trends towards improvements in visual memory and spatial plannins abilities

No significant improvements in executive functions, procedural memory (SOC), sustained attention (RVP), semantic memory (PAL) and working memory (SWM)

Impact of quetiapine on cognition; 12-week open-label trial

Improvements were noticed on the PAL and SWM tasks; authors were not able to rule out a practice effect

Investigated the potential cognitive toxicity of quetiapine prescribed at dosage higher than the recommended (200-800 mg); for 6 weeks
Observed no decline in psychomotor speed and spatial working memory; suggesting a lack of cognitive toxicity requires revised treatment regimen

Note: For abbreviation of neuropsychological tasks refer to Table 1. DUP, duration of untreated psychosis; SUD, substance use disorder; LI, latent inhibition.

\section{Discussion}

Cognitive dysfunction has been recognized as a primary and enduring core deficit in schizophrenia, rather than the previous focus on positive and negative symptoms. Schizophrenia patients have difficulties performing tests of attention, working memory, verbal learning and memory, executive functions, language skills, and social 
cognition. These deficits are linked to many functional domains, including impairments in occupational and social behavior. However, the study of cognition in schizophrenia raises practical and methodological concerns, which have led researchers to use a broad range of tests and batteries, including CANTAB. Over the last decade, this battery has been regularly utilized in cognitive studies in schizophrenia. In this review, we have evaluated the strengths and weaknesses of CANTAB for the assessment of cognition in schizophrenia, as the battery is the subject of more and more interest.

The use of a computerized battery presents some methodological and practical advantages over traditional neuropsychological tests. The main advantage of CANTAB is the standardization of administration. The rigorous experimental heritage of the CANTAB and the wide range of data already collected have allowed effects to be related to particular components of cognitive function and to potential underlying neural substrates. In the aftermath of these results, neuroimaging studies have been initiated with the promise of a greater understanding of the neurobiological bases of neurological and psychiatric disorders.

Several neuropsychologists and researchers remain skeptical about this new era of computerized testing, mainly because of the lack of comparison between traditional neuropsychological tests and these new computerized tests, including CANTAB. At the moment, clear guidelines on how to use CANTAB for assessment of change in cognitive function are lacking. Thus, CANTAB alone cannot substitute for the traditional neuropsychological tests routinely used in clinical settings. In addition, the ecological validity of CANTAB needs to be further investigated in order to establish the value of CANTAB tests as predictors of community functioning. Furthermore, this battery necessitates methodological improvements, such as standardization with young populations. According to Luciana [32], CANTAB is valid in populations ranging from 4 to 90 years of age. However, it remains to be shown whether CANTAB is also valid in schizophrenia patients for the same age range.

Despite these shortcomings, the use of CANTAB in schizophrenia has generally corroborated results of neuropsychological studies using traditional tests and has allowed an advance in our knowledge about cognition in schizophrenia. For instance, CANTAB has enabled researchers to highlight significant deficits affecting broad cognitive domains in schizophrenia (e.g. working memory, decision-making, attention, executive functions, and visual memory). The cognitive deficits highlighted in schizophrenia with CANTAB are similar to those documented using traditional (visuo-spatial) neuropsychological tests (e.g., Tower of London, WCST) [13].

This pattern of cognitive impairments in schizophrenia shows great variability from one patient to another. This heterogeneity is plausible in light of the symptomatic heterogeneity of schizophrenia. In the last decade, important efforts have been invested in providing a more refined description of the phenotypic features of the pathology, using neuropsychological tests [27]. From that perspective, CANTAB tasks have been recently used to divide schizophrenia patients into distinctive cognitive profiles subgroups, both empirically and clinically useful. Thus far, CANTAB has been successfully used to distinguish the cognitive profile of: (i) schizophrenia patients with mild (type A) and marked (type B) neurological signs [2]; schizophrenia patients with and without substance use disorder [45]; and (iii) patients with schizophrenia versus schizoaffective disorder [62]. In light of cognitive remediation, knowledge of differences between cognitive profiles is very important in order to facilitate revalidation to particular patients. Although encouraging, these results must be replicated by other research teams before it can be concluded that CANTAB is useful for schizophrenia's cognitive profile subtyping.

Preliminary evidence suggests that CANTAB may be a useful tool to identify cognitive dysfunctions predicting psychosis. Wood et al. [67] have showed impairments in working memory and executive functions in young people at ultrahigh risk of developing psychosis, relative to controls. Similarly, Bartok et al. [7] have described overall deficits in memory and attention in young adults at the prodromal stage of psychosis, relative to controls. Further studies to validate the use of CANTAB as a predictive tool for early detection of individuals at risk of developing psychosis are warranted.

CANTAB has been successfully used to compare the cognitive profile of schizophrenia patients, relative to the cognitive performance of patients with other acquired pathologies, including patients with unipolar depression [11], frontal lobe lesions and basal ganglia dysfunctions [43], PD and temporal lobe lesions [43], Alzheimer's disease [16], and bipolar disorder [35]. Interestingly, studies comparing schizophrenia patients to other patient groups using CANTAB have consistently highlighted deficits in executive functions, coherent with frontostriatal dysfunctions in the disorder. Functional brain imaging studies are required to corroborate this pathophysiological assumption. 
Also relevant to the pathophysiology of schizophrenia, preliminary studies have showed positive correlations between occulomotor frontal cognitive deficits in schizophrenia, using CANTAB [21,22]. Although preliminary, the relationships described in these studies are consistent with the results of studies using traditional neuropsychological tests [29]. In addition, these results suggest that cognitive and occulomotor deficits in schizophrenia may share common abnormal frontal substrates.

CANTAB has also been used to explore the associations between visuo-spatial cognition and community functioning. Baseline performance in attention and working memory, which are impaired early in schizophrenia, predicted improvements in community functioning and behavioral problems, such as medication compliance. In addition, planning abilities, which are also disturbed at the onset of the disease, predicted improvements in social competence $[48,49]$. The fact that cognition, as measured with CANTAB, predicted psychosocial functioning is consistent with the results obtained in studies using traditional neuropsychological tests [18]. Further studies are needed to validate the use of CANTAB to identify cognitive predictors of social functioning.

As for the use of CANTAB in the evaluation of the impacts of pharmacological agents on cognition in schizophrenia, a few promising studies have been conducted so far. However, their validity is questioned by the lack of studies comparing the impact of medications on cognition, as measured with traditional neuropsychological tests and CANTAB tasks. It remains to be determined if CANTAB is sensitive enough to measure the cognitive impact of pharmacological interventions.

CANTAB comprises "user-friendly" computerized visuo-spatial tasks administered in a standardized way, which will require, in the future, to be compared head-to-head to traditional neuropsychological tests. In schizophrenia, this battery has been successfully used in cross-sectional studies, which have highlighted a broad range of visuospatial cognitive deficits in schizophrenia, consistent with the results obtained with some traditional neuropsychological tests, like the Tower of London and the WCST. However, we must draw prudent conclusions in regards to usability of CANTAB in assessment of the whole visuo-spatial cognitive deficit in schizophrenia, due to the fact that most of studies reviewed herein tested only a subset of visuo-spatial abilities.

\section{References}

[1] Almeida OP, Howard RJ, Levy R, David AS, Morris RG, Sahakian BJ. Cognitive features of psychotic states arising in late life (late paraphrenia). Psychol Med 1995;25(4):685-98.

[2] Almeida OP, Howard RJ, Levy R, David AS, Morris RG, Sahakian BJ. Clinical and cognitive diversity of psychotic states arising in late life (late paraphrenia). Psychol Med 1995;25(4):699-714.

[3] Badcock JC, Michiel PT, Rock D. Spatial working memory and planning ability: contrasts between schizophrenia and bipolar I disorder. Cortex 2005;41(6):753-63.

[4] Baker SC, Rogers RD, Owen AM, Frith CD, Dolan RJ, Frackowiak RS, et al. Neural systems engaged by planning: a PET study of the Tower of London task. Neuropsychologia 1996;34(6):515-26.

[5] Barnes TR, Hutton SB, Chapman MJ, Mutsatsa S, Puri BK, Joyce EM. West London first-episode study of schizophrenia. Clinical correlates of duration of untreated psychosis. Br J Psychiatry 2000;177:207-11.

[6] Barnes TR, Mutsatsa SH, Hutton SB, Watt HC, Joyce EM. Comorbid substance use and age at onset of schizophrenia. Br J Psychiatry 2006;188:237-42

[7] Bartok E, Berecz R, Glaub T, Degrell I. Cognitive functions in prepsychotic patients. Prog Neuropsychopharmacol Biol Psychiatry 2005;29(4): 621-5.

[8] Berg E. A simple objective technique for measuring flexibility in thinking. J Gen Psychol 1948;39:15-22.

[9] Chouinard S, Poulin J, Stip E, Godbout R, Lalonde P, Melun JP, et al. Cognitive performance in patients with schizophrenia after rivastigmine treatment. XX International Congress on Schizophrenia Research. Schizophr Bull April 2005;2005:478.

[10] Elliott R, McKenna PJ, Robbins TW, Sahakian BJ. Neuropsychological evidence for frontostriatal dysfunction in schizophrenia. Psychol Med 1995;25(3):619-30.

[11] Elliott R, McKenna PJ, Robbins TW, Sahakian BJ. Specific neuropsychological deficits in schizophrenic patients with preserved intellectual function. Cognit Neuropsychiatry 1998;3(3):45-70.

[12] Elliott R, Sahakian BJ, McKay AP, Herrod JJ, Robbins TW, Paykel ES. Neuropsychological impairments in unipolar depression: the influence of perceived failure on subsequent performance. Psychol Med 1996;26(5): 975-89. 
[13] Elliott R, Sahakian BJ. The neuropsychology of schizophrenia: relations with clinical and neurobiological dimensions. Psychol Med 1995;25(3): 581-94.

[14] Fowler KS, Saling MM, Conway EL, Semple JM, Louis WJ. Paired associate performance in the early detection of DAT. J Int Neuropsychol Soc 2002;8(1):58-71.

[15] Fray PJ, Robbins TW, Sahakian BJ. Neuropsychiatric applications of CANTAB. Int J Geriatr Psychiatry 1996;11(4):329-36.

[16] Gabrovska-Johnson VS, Scott M, Jeffries S, Thacker N, Baldwin RC, Burns A, et al. Right-hemisphere encephalopathy in elderly subjects with schizophrenia: evidence from neuropsychological and brain imaging studies. Psychopharmacology (Berl) 2003;169(3-4):36775 .

[17] Gaffan D. Recognition impaired and association intact in the memory of monkeys after transection of the fornix. J Comp Physiol Psychol 1974;86(6):1100-9.

[18] Green MF, Kern RS, Heaton RK. Longitudinal studies of cognition and functional outcome in schizophrenia: implications for MATRICS. Schizophr Res 2004;72(1):41-51.

[19] Green MF, Nuechterlein KH. The MATRICS initiative: developing a consensus cognitive battery for clinical trials. Schizophr Res 2004; 72(1):1-3.

[20] Hamilton JM, Salmon DP, Corey-Bloom J, Gamst A, Paulsen JS, Jerkins S, et al. Behavioural abnormalities contribute to functional decline in Huntington's disease. J Neurol Neurosurg Psychiatry 2003;74(1): 120-2.

[21] Hutton S, Huddy. The relationship between antisaccades, smooth pursuit, and executive dysfunction in first-episode schizophrenia. Biol Psychiatry 2004;56(8):553-9.

[22] Hutton SB, Cuthbert I, Crawford TJ, Kennard C, Barnes TR, Joyce EM. Saccadic hypometria in drug-naive and drug-treated schizophrenic patients: a working memory deficit? Psychophysiology 2001;38(1): 125-32.

[23] Hutton SB, Huddy V, Barnes TR, Robbins TW, Crawford TJ, Kennard C, et al. The relationship between antisaccades, smooth pursuit, and executive dysfunction in first-episode schizophrenia. Biol Psychiatry 2004;56(8):553-9.

[24] Hutton SB, Murphy FC, Joyce EM, Rogers RD, Cuthbert I, Barnes TR, et al. Decision making deficits in patients with first-episode and chronic schizophrenia. Schizophr Res 2002;55(3):249-57.

[25] Hutton SB, Puri BK, Duncan LJ, Robbins TW, Barnes TR, Joyce EM. Executive function in first-episode schizophrenia. Psychol Med $1998 ; 28(2): 463-73$

[26] Joyce E, Hutton S, Mutsatsa S, Gibbins H, Webb E, Paul S, et al. Executive dysfunction in first-episode schizophrenia and relationship to duration of untreated psychosis: the West London Study. Br J Psychiatry Suppl 2002;43:s38-44.

[27] Joyce EM, Hutton SB, Mutsatsa SH, Barnes TR. Cognitive heterogeneity in first-episode schizophrenia. Br J Psychiatry 2005;187:51622

[28] Joyce EM, Robbins TW. Frontal lobe function in Korsakoff and non-Korsakoff alcoholics: planning and spatial working memory. Neuropsychologia 1991;29(8):709-23

[29] Karoumi B, Ventre-Dominey J, Vighetto A, Dalery J, d'Amato T Saccadic eye movements in schizophrenic patients. Psychiatry Res 1998;77(1):9-19.

[30] Lawrie SM, Ingle GT, Santosh CG, Rogers AC, Rimmington JE, Naidu KP, et al. Magnetic resonance imaging and single photon emission tomography in treatment-responsive and treatment-resistant schizophrenia. Br J Psychiatry 1995;167(2):202-10.

[31] Lowe C, Rabbitt P. Test/re-test reliability of the CANTAB and ISPOCD neuropsychological batteries: theoretical and practical issues. Cambridge Neuropsychological Test Automated Battery. International Study of Post-Operative Cognitive Dysfunction. Neuropsychologia $1998 ; 36(9): 915-23$.

[32] Luciana M. Practitioner review: computerized assessment of neuropsychological function in children: clinical and research applications of the Cambridge Neuropsychological Testing Automated Battery (CANTAB). J Child Psychol Psychiatry 2003;44(5):649-63.

[33] Mathes B, Wood SJ, Proffitt TM, Stuart GW, Buchanan JA, Velakoulis D, et al. Early processing deficits in object working memory in first-episode schizophreniform psychosis and established schizophrenia. Psychol Med 2005;35(7): 1053-62.

[34] McCartan D, Bell R, Green JF, Campbell C, Trimble K, Pickering A, et al. The differential effects of chlorpromazine and haloperidol on latent inhibition in healthy volunteers. J Psychopharmacol 2001;15(2):96-104.

[35] Mcintosh AM, Harrison LK, Forrester K, Lawrie SM, Johnstone EC. Neuropsychological impairments in people with schizophrenia or bipolar disorder and their unaffected relatives. Br J Psychiatry 2005; 186: 378-85. 
[36] Milner B. Interhemispheric differences in the localization of psychological processes in man. Br Med Bull 1971;27(3):272-7.

[37] Mishkin M. A memory system in the monkey. Philos Trans R Soc Lond B Biol Sci 1982;298(1089):83-95.

[38] Owen AM, Evans AC, Petrides M. Evidence for a two-stage model of spatial working memory processing within the lateral frontal cortex: a positron emission tomography study. Cereb Cortex 1996;6(1):31-8.

[39] Owen AM, Morris RG, Sahakian BJ, Polkey CE, Robbins T. Double-dissociation of memory and executive functions in working memory tasks following frontal-lobe excisions, temporal lobe excisions or amygdalo-hippocampectomy in man. Brain 1997;119(5):1597615.

[40] Owen AM, Sahakian BJ, Semple J, Polkey CE, Robbins TW. Visuospatial short-term recognition memory and learning after temporal lobe excisions, frontal lobe excisions or amygdalo-hippocampectomy in man. Neuropsychologia 1995;33(1):1-24.

[41] Pampoulova T, Potvin S, Sepehry AA, Levaux MN, Feltrin CES. Lack of cognitive toxicity during high-dose quetiapine therapy. Schizophr Res 2006;81(Suppl S):138.

[42] Pantelis C, Barber FZ, Barnes TR, Nelson HE, Owen AM, Robbins TW. Comparison of set-shifting ability in patients with chronic schizophrenia and frontal lobe damage. Schizophr Res 1999;37(3):251-70.

[43] Pantelis C, Barnes TR, Nelson HE, Tanner S, Weatherley L, Owen AM, et al. Frontal-striatal cognitive deficits in patients with chronic schizophrenia. Brain 1997;120(Pt 10):1823-43.

[44] Pantelis C, Stuart GW, Nelson HE, Robbins TW, Barnes TR. Spatial working memory deficits in schizophrenia: relationship with tardive dyskinesia and negative symptoms. Am J Psychiatry 2001;158(8):1276-85.

[45] Potvin S, Briand C, Prouteau A, Bouchard RH, Lipp O, Lalonde P, et al. CANTAB explicit memory is less impaired in addicted schizophrenia patients. Brain Cogn 2005;59(1):38-42.

[46] Potvin S, Briand C, Prouteau A, Bouchard RH, Lipp O, Lalonde P, et al. CANTAB explicit memory is less impaired in addicted schizophrenia patients. Brain Cogn 2005;59(1):38-42.

[47] Potvin S, Stip E, Lipp O, Mancini-Marie A, Elie R, Demers MF, et al. Quetiapine in patients with comorbid schizophrenia-spectrum and substance use disorders: an open-label trial. Curr Med Res Opin 2006; 22(7): 1277-85.

[48] Prouteau A, Verdoux H, Briand C, Lesage A, Lalonde P, Nicole L, et al. The crucial role of sustained attention in community functioning in outpatients with schizophrenia. Psychiatry Res 2004;129(2):171-7.

[49] Prouteau A, Verdoux H, Briand C, Lesage A, Lalonde P, Nicole L, et al. Cognitive predictors of psychosocial functioning outcome in schizophrenia: a follow-up study of subjects participating in a rehabilitation program. Schizophr Res 2005;77(2-3):343-53.

[50] Prouteau A, Verdoux H, Briand C, Lesage A, Lalonde P, Nicole L, et al. Self-assessed cognitive dysfunction and objective performance in outpatients with schizophrenia participating in a rehabilitation program. Schizophr Res 2004;69(1):85-91.

[51] Regier DA, Farmer ME, Rae DS, Locke BZ, Keith SJ, Judd LL, et al. Comorbidity of mental disorders with alcohol and other drug abuse. Results from the Epidemiologic Catchment Area (ECA) Study. JAMA 1990;264(19):2511-8.

[52] Ritsner MS, Blumenkrantz H, Dubinsky T, Dwolatzky T. The detection of neurocognitive decline in schizophrenia using the Mindstreams Computerized Cognitive Test Battery. Schizophr Res 2006;82(1):39-49.

[53] Robbins TW, James M, Owen AM, Sahakian BJ, Mclnnes L, Rabbitt P. Cambridge Neuropsychological Test Automated Battery (CANTAB): a factor analytic study of a large sample of normal elderly volunteers. Dementia 1994;5(5):266-81.

[54] Robbins TW. Dissociating executive functions of the prefrontal cortex. Philos Trans R Soc Lond B Biol Sci 1996;351(1346):1463-70 (discussion 70-1).

[55] Robbins TW. The case of frontostriatal dysfunction in schizophrenia. Schizophr Bull 1990;16(3):391-402.

[56] Rosvald HE, Mirsky AF, Sarason I, Bransome EDJ, Beck LD. A continuous performance test of brain damage. J Consult Psychol 1956;20(5): 343-50.

[57] Sahakian BJ, Coull JJ, Hodges JR. Selective enhancement of executive function by idazoxan in a patient with dementia of the frontal lobe type. J Neurol Neurosurg Psychiatry 1994;57(1):120-1.

[58] Sahakian BJ, Morris RG, Evenden JL, Heald A, Levy R, Philpot M, et al. A comparative study of visuospatial memory and learning in Alzheimer-type dementia and Parkinson's disease. Brain 1988;111(Pt 3):695-718.

[59] Sahgal A, Sahakian BJ, Robbins TW, Wray CJ. Detection of visual memory and learning deficits in Alzheimer's disease using the Cambridge Neuropsychological Test Automated Battery. Dementia 1991;2(3): 150-8. 
Published in: European Psychiatry (2007), vol. 22, pp. 104-115.

Status: Postprint (Author's version)

[60] Shallice T. Specific impairments of planning. Philos Trans R Soc Lond B Biol Sci 1982;298(1089): 199-209.

[61] Squire LR. Memory and the hippocampus: a synthesis from findings with rats, monkeys, and humans. Psychol Rev 1992;99(2): 195231 .

[62] Stip E, Sepehry AA, Prouteau A, Briand C, Nicole L, Lalonde P, et al. Cognitive discernible factors between schizophrenia and schizoaffective disorder. Brain Cogn 2005;59(3):292-5.

[63] Strauss E, Sherman EMS, Spreen O. CANTAB. In: A compendium of neuropsychological tests: administration, norms, and commentary. $3^{\text {rd }}$ ed. New York: Oxford University Press; 2006. p. 415-24.

[64] Turner DC, Clark L, Pomarol-Clotet E, McKenna P, Robbins TW, Sahakian BJ. Modafinil improves cognition and attentional set shifting in patients with chronic schizophrenia. Neuropsychopharmacology 2004;29(7):1363-73.

[65] Tyson PJ, Laws KR, Roberts KH, Mortimer AM. A longitudinal analysis of memory in patients with schizophrenia. J Clin Exp Neuropsychol 2005;27(6):718-34

[66] Tyson PJ, Laws KR, Roberts KH, Mortimer AM. Stability of set-shifting and planning abilities in patients with schizophrenia. Psychiatry Res 2004;129(3):229-39.

[67] Wood SJ, Pantelis C, Proffitt T, Phillips LJ, Stuart GW, Buchanan JA, et al. Spatial working memory ability is a marker of risk-forpsychosis. Psychol Med 2003;33(7):1239-47. 